\title{
The Prebiotic Effect of Triple Biotic Technology on Skin Health
}

\author{
Min Li*, Aixing Fan\#, Junhong Mao\#, Nadia Soliman, Komal Shahani, Andre' M. Morgan, \\ Thomas Boyd
}

Colgate-Palmolive Company, Technology Center, Piscataway, New Jersey, USA

Email: ^Min_li@colpal.com

How to cite this paper: Li, M., Fan, A.X., Mao, J.H., Soliman, N., Shahani, K., Morgan, A.M. and Boyd, T. (2021) The Prebiotic Effect of Triple Biotic Technology on Skin Health. Journal of Cosmetics, Dermatological Sciences and Applications, 11, 304-319.

https://doi.org/10.4236/jcdsa.2021.114025

Received: September 9, 2021

Accepted: December 4, 2021

Published: December 7, 2021

Copyright () 2021 by author(s) and Scientific Research Publishing Inc. This work is licensed under the Creative Commons Attribution International License (CC BY 4.0).

http://creativecommons.org/licenses/by/4.0/

\section{(c) (i) Open Access}

\begin{abstract}
Objective: Pre-, pro- and postbiotics are becoming more prevalent as ingredients in cosmetic and personal care products. A novel triple biotic technology has been developed and investigated for its impact on skin flora and skin barrier properties. Methods: Growth inhibition/promotion assay was performed to determine the effect on skin bacteria growth, using Escherichia coli, Corynebacterium striatum, Staphylococcus aureus, and Staphylococcus epidermidis. A skin penetration assay and skin barrier biomarker measurements were performed using an ex vivo human skin explant model. The triple-biotic complex of inulin, 2-butyloctanol, and a biomimic blend of postbiotics was tested individually as well as part of cosmetic formulations. Results: The triple-biotic technology, either as individual components or in a cosmetic formulation, inhibited the growth of undesirable bacteria, in most cases. On the other hand, the growth of desirable bacteria was either promoted or maintained. The cosmetic formulations with the triple-biotic technology demonstrated an enhanced skin barrier and an increase in skin barrier biomarkers. Conclusion: A novel triple-biotic technology has been developed and shown to deliver a strong prebiotic effect with demonstrable benefits on bacterial growth, skin barrier properties, and the production of skin barrier biomarkers. This study indicates that triple-biotic technology can be used as a desirable prebiotic ingredient in personal care products to provide skin health benefits.
\end{abstract}

\section{Keywords}

Triple Biotic Technology, Prebiotics, Postbiotics, Skin Microbiome, Skin Barrier 


\section{Introduction}

The nature of skincare and the cosmetic/personal care industry is rapidly changing with respect to the inclusion of new and novel constituents. Once found strictly in dietary supplements and other nutrient-rich sources, prebiotics and probiotics are becoming more prevalent as ingredients in cosmetic and personal care products. A prebiotic is a "substrate that is selectively utilized by microorganisms conferring a health benefit" [1] [2]. There have been a number of recent articles that have reviewed the impact of prebiotics on skin health [3] [4] [5] [6] [7]. In their review on the impact of prebiotics and probiotics on skin health, Al-Ghazzewi and Tester reported that prebiotics, when applied directly to skin, can selectively increase the activity and growth of beneficial skin microbiota. However, they further stated that little is known about the overall efficacy of this prebiotics [3]. Ouwehand et al. concluded in their review that prebiotics had been shown to improve skin health and provide relief to persons with atopic dermatitis [7]. In addition, a clinical study conducted in patients with atopic dermatitis concluded that the use of a topically applied prebiotic was a novel therapeutic approach that could be used to enable normalization of skin microbiota. Many of these articles have focused on the impact of a single prebiotic due to the potential for more than one pre or probiotics to have interactions or increase the complexity of the study.

Plant-based ingredients are commonly used in cosmetics and personal care products. One such ingredient, inulin, is safe and effective [8]. Inulin and inulin-type fructans and galacto-oligosaccharides have demonstrated prebiotic effects in a number of ways, but little is known about its prebiotic effect on skin health when applied topically [9] [10] [11]. Recently, we discovered another cosmetic ingredient, 2-butyloctanol to have a prebiotic effect on the human axillary microbiome by inhibiting undesirable odor-causing corynebacteria while maintaining/promoting skin-friendly staphylococcus [12].

Conversely, probiotics are living microorganisms, which confer a health benefit on the host when administered in adequate amounts [13]. It is difficult, if not impossible, to formulate a cosmetic product using living microorganisms and have them be active throughout the shelf life of the product. Only a few companies have incorporated probiotics into their products [14]. Researchers have gotten around this limitation by using killed probiotics, or postbiotics, which are the byproducts of the fermentation process produced by probiotics, in the product [15]. There are limited examples of topical applications. Guéniche et al. have shown Bifidobacterium longum sp. extract, formulated at the $10 \%$ level in a topical cream, has the potential of decreasing skin reactivity and improving skin barrier function [16]. Another example is the lactobacilli group of bacteria, which have been used successfully as a wound treatment [17]. Lactobacillus rhamnosus is a well-known probiotics. It has been shown that oral administration of tyndallized Lactobacillus rhamnosus successfully treated atopic dermatitis [18], and a postbiotic derived from Lactobacillus rhamnosus GG has a beneficial effect on intestinal barrier function [19]. However, little is known about its prebiotic effect 
on skin health topically.

Thus, a triple biotic complex of inulin, 2-butyloctanol, and a biomimic blend of postbiotics, lactic acid and pyruvic acid, that were isolated from Lactobacillus rhamnosus fermented with xylitol, has been developed and incorporated into a body wash and a body lotion. These individual ingredients as well products have been compared to their respective placebos in a series of tests that have been designed to evaluate the prebiotic effect of this triple biotic technology.

\section{Materials and Methods}

\subsection{Triple Biotic Technology Development}

The triple biotic technology consists of three key actives: inulin, 2-butyloctanol, and a biomimic blend of postbiotics. The biomimic postbiotic blend was generated from the key short chain fatty acids isolated from the fermentation of Lactobacillus rhamnosus fed with xylitol.

A pure colony of L. rhamnosus LR 32 (Dupont, Wilmington, Delaware) was grown in MRS medium in $5 \% \mathrm{CO}_{2}$ incubator at $37^{\circ} \mathrm{C}$ overnight. The turbidity of the lactobacillus solution was adjusted to Optical Density $=0.1(\mathrm{OD})$ at $610 \mathrm{~nm}$ using a UV-VIS Spectrometer (Lambda ${ }^{\mathrm{TM}} 45$, PerkinElmer Inc, Waltham, Massachusetts, USA). Four milliliters of lactobacillus culture $(\mathrm{OD}=0.1)$ was added into $16 \mathrm{ml}$ of MRS medium with $2.5 \%$ xylitol, and incubated in $5 \% \mathrm{CO}_{2}$ at $37^{\circ} \mathrm{C}$ overnight. Next day, the turbidity of the lactobacillus culture was adjusted to OD $=1$ using sterile distilled water, then centrifuged $12,000 \mathrm{rpm}$ for $10 \mathrm{~min}$. The supernatant was collected and then passed through a $0.22 \mu \mathrm{m}$ filter. The lactobacillus ferment was stored at $4^{\circ} \mathrm{C}$ for further usage.

The short chain fatty acids in lactobacillus ferment were analyzed by GC-MS at NIZO food research (Ede, The Netherlands). Lactic acid and pyruvic acid were detected as the main short chain fatty acids. A blend of lactic acid and pyruvic acid at the ratio of 4:1 was generated according to the GC-MS profile, called biomimic postbiotic blend. Sodium pyruvate was used in the formulation to replace pyruvic acid due to hazardous status and issue in shipping.

\subsection{In Vitro Micro Test Method}

Growth inhibition/promotion assay was performed to determine whether the active or formula inhibit/promote bacteria growth. The test bacteria (VWR, Radnor, PA, USA) were Escherichia coli (ATCC 11229), Corynebacterium striatum (ATCC 1293), Staphylococcus aureus (ATCC 6538), and Staphylococcus epidermidis (ATCC 12228).

Solutions of inulin (5\%) (The Iidea Company, Jalisco, México) and the biomimic blend (0.6\%) were prepared in Tryptic Soy Broth (TSB) medium. A pure colony of individual test bacteria was grown in TSB medium overnight. The turbidity of the bacterial culture solution was adjusted to $\mathrm{OD}=0.1$ at $610 \mathrm{~nm}$ using a UV-VIS Spectrometer. Then $2 \mathrm{ml}$ of the bacteria culture solution was incubated with $2 \mathrm{ml}$ of test neat ingredient at $37^{\circ} \mathrm{C}$ for $48 \mathrm{~h}$. The bacteria incubated 
with medium alone were considered the control. The optical density of each tube was read after incubation using a UV-VIS Spectrometer. Each treatment was evaluated in triplicates.

Test body wash or body lotion products with triple biotic technology as well as their respective placebos (Colgate-Palmolive Company, New York, NY, USA) were diluted to $1 \%$ solution in TSB medium. Pure individual colonies of the selected bacteria were grown in TSB medium overnight. The turbidity of the bacterial culture solutions was adjusted to $\mathrm{OD}=0.1$ at $610 \mathrm{~nm}$ using a UV-VIS Spectrometer. Following this, $2 \mathrm{ml}$ of each bacteria culture solution was incubated with $2 \mathrm{ml}$ of a $1 \%$ solution each test sample at $37^{\circ} \mathrm{C}$ for 3 hours. After incubation, each solution was serially diluted 10 times in TSB. A sample of $100 \mu \mathrm{l}$ of the solution was plated on Tryptic Soy Agar (TSA) plates and incubated at $37^{\circ} \mathrm{C}$ overnight. The bacterial colonies were counted the next day. Test samples were evaluated in two separate experiments with three replicates each. The data were expressed as $\log 10$ colony-forming units $(\mathrm{CFU}) / \mathrm{ml}$. The ratio of desirable bacteria to undesirable bacteria was calculated by dividing the log counts of desirable bacteria by the log counts of undesirable bacteria.

\subsection{Ex Vivo Skin Test Methods}

The ex vivo tests were performed by the Padova laboratories of Symrise srl, Milan, Italy.

\subsubsection{Skin Preparation}

The skin explants were obtained from patients undergoing abdominal plastic surgery, cut into pieces of approximately $8 \mathrm{~mm} \times 3 \mathrm{~mm}$ thickness and cultured for three days for the Skin Penetration Assay and for six days for the Skin Barrier Biomarker Measurements.

\subsubsection{Application of Body Washes}

The skin tissues were gently cleaned with a cotton pad. Afterwards, $4 \mu \mathrm{l}$ of a $10 \%$ diluted solution for the Test and Placebo body washes was applied on top of each tissue, covered with a $6 \mathrm{~mm}$ delivery membrane, and left in place for $15 \mathrm{~min}$. Afterwards the skin tissues were gently cleaned until the next application. The application process was performed daily. The tissues were collected at day 3 for the skin penetration assay.

\subsubsection{Application of Body Lotions}

The skin tissues were gently cleaned with a cotton pad. Afterwards, $4 \mu \mathrm{l}$ of the Test and Placebo body lotions was applied on top of each tissue, covered with a 6 $\mathrm{mm}$ delivery membrane, and left in place for $24 \mathrm{~h}$. The application process was repeated daily. The tissues were collected at day 3 for the skin penetration assay and day 6 for skin barrier biomarker measurements.

\subsection{Skin Penetration Assay}

Skin tissues (6) were harvested, stained with Rhodamine B, cryo-fixed, and cut 
by the cryostat for subsequent image acquisition and analysis. Analysis of Rhodamine B fluorescence (red color) was performed within the epidermis area using Image-J (NIH, Bethesda, MD, USA). For each skin tissue, two sections were selected; fluorescent images acquired, and analyzed for a total of 12 data points. $(6$ skin samples $\times 2$ sections $=12$ sampling points/data for each tested condition). The values were normalized using the dimension of the selected sections.

\subsection{Skin Barrier Biomarker Measurements}

Two sections from each of six skin tissues were immunostained with the selected antibody for Filaggrin (SantaCruz Biotechnology, Dallas, TX, USA), Involucrin, Desmocollin 1 and Claudin 1 (Abcam, Cambridge, UK). The amount of the antigen present in each slide was evaluated by estimating the intensity and distribution of the pink/red within the epidermis using Image J (NIH, Bethesda, MD, USA). The values were normalized on the dimension of the analyzed surface expressed in pixels.

\section{Statistics}

The mean value and standard deviation of optical density or bacteria counts in each tested products were calculated in excel. The statistical significance between the effects of the test products on bacterial optical density or counts was analyzed by one-way ANOVA at the $95 \%$ confidence level using Minitab software (Version 17, State College, PA, USA).

For the ex vivo test, all quantitative data were summarized in terms of the mean score for each treatment. The measures of variation as standard deviation were applied to the original scores. Statistical significances between groups were evaluated by one-way ANOVA with permutation at the 95\% confidence level followed by $\mathrm{t}$-test with permutation using B1SCLASSIC Software (SISSAD snc, Trieste, Italy).

\section{Results}

The impact of triple biotic technology on skin bacteria growth was investigated individually and in a cosmetic formulation using growth/inhibition assay.

For neat ingredients, in comparison to the control solution, the 5\% solution of inulin (Figure 1) significantly inhibited the growth of E. coli, C. striatum, and $S$. aureus, while maintaining the growth of $S$. epidermidis. The $0.6 \%$ solution of the biomimic blend (Figure 2) significantly inhibited the growth of $E$. coli and $C$. striatum, while maintaining the growth of $S$. aureus. Conversely, it significantly promoted the growth of $S$. epidermidis.

2-butyloctanol works as a smart biotic component in the triple biotic technology. 2-butyloctanol has been shown to preferably inhibit odor causing corynebacterium while maintaining/promoting skin friendly staphylococcus in both in vitro and in vivo studies in our previous study [12]. 


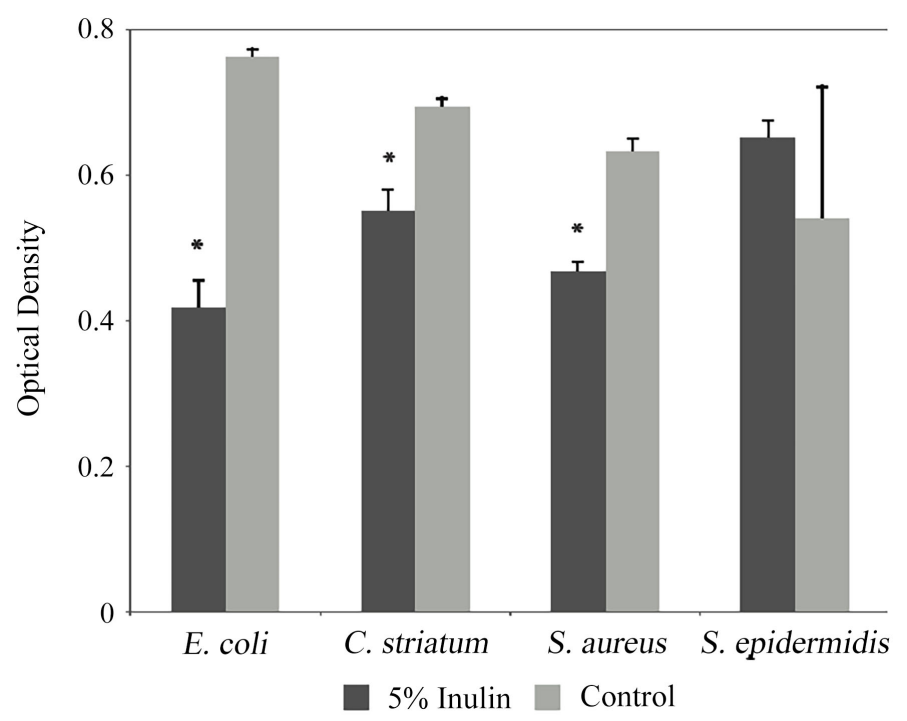

Figure 1. Prebiotic Effect of Inulin (5\% solution) on skin bacteria. Inulin (5\%) inhibits undesirable bacteria growth $(E$. coli, $C$. striatum, $S$. aureus) and maintains desirable bacterial growth $\left(S\right.$. epidermidis) in vitro. ${ }^{*}$ significant difference compared to control $(\mathrm{p}<$ $0.05)$.

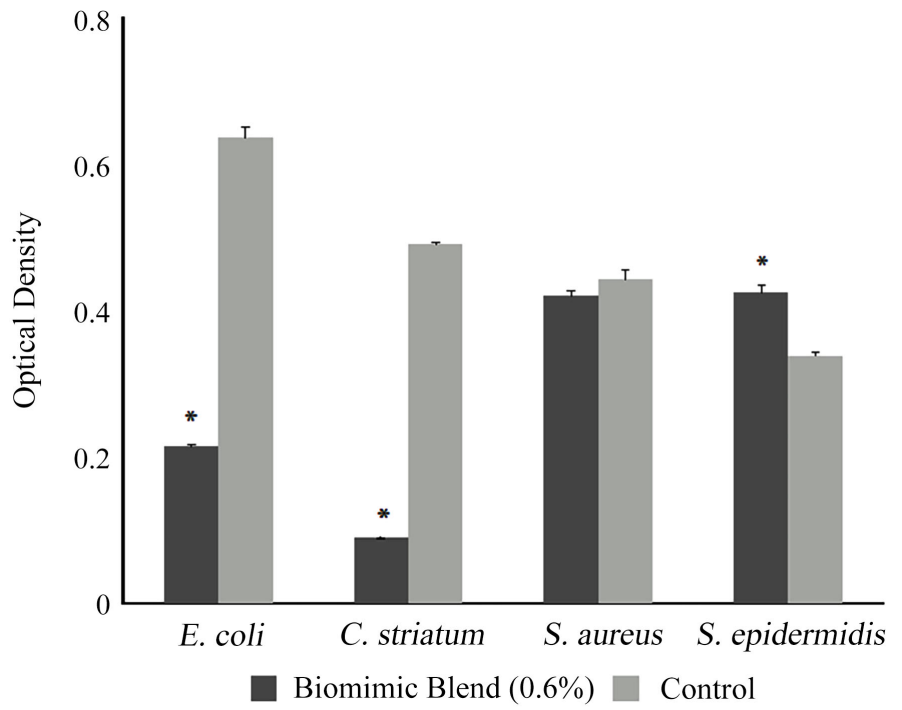

Figure 2. Prebiotic Effect of Biomimic Blend (0.6\% solution) on skin bacteria. Biomimic Blend $(0.6 \%)$ inhibits growth of undesirable bacteria growth (E. coli, $C$. striatum), maintains growth of undesirable bacteria $(S$. aureus) and promotes growth of desirable bacteria $\left(S\right.$. epidermidis) in vitro. ${ }^{\star}$ significant difference compared to control $(\mathrm{p}<0.05)$.

In comparison to the Placebo Body Wash, the Test Body Wash with the triple biotic technology demonstrated a prebiotic effect by inhibiting the growth of $C$. striatum and maintaining the growth of $S$. epidermidis (Figure 3(a) and Figure $3(\mathrm{~b}))$. These results, expressed in $\log 10 \mathrm{CFU} / \mathrm{ml}$, show a statistically significant difference between the two body washes on $C$. striatum $(\mathrm{p}=0.042)$ and non-statistical tendency on $S$. epidermidis $(\mathrm{p}=0.052)$. In addition, as seen in Figure $3(c)$, the Test Body Wash has statistically significantly increased the ratio 


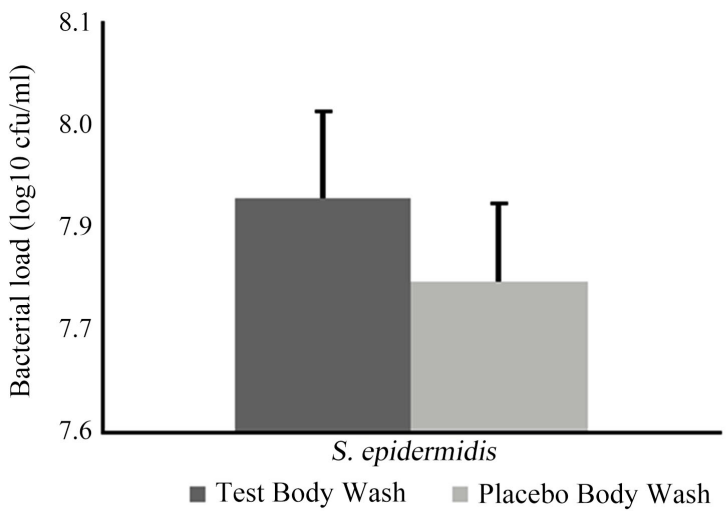

(a)

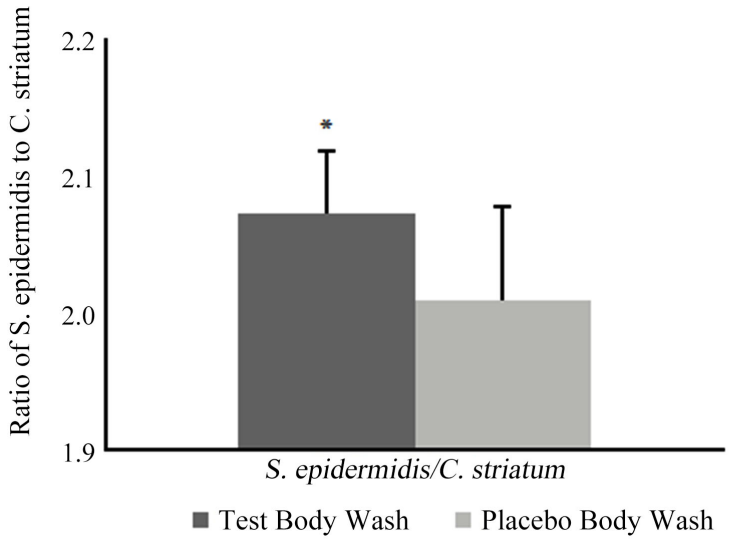

(c)

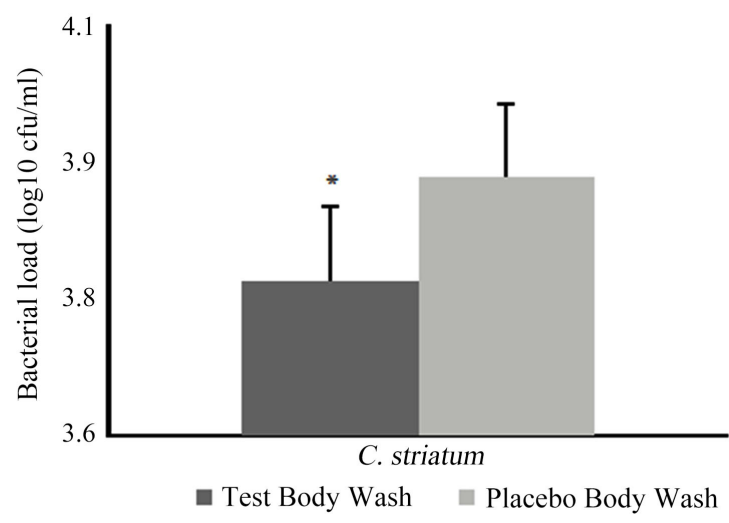

(b)

Figure 3. Comparison of Body Wash with and without Triple Biotics Technology of the growth of S. epidermidis (3a), C. striatum (3b), and the ratio of $S$. epidermidis/C. striatum (3c). ${ }^{\star}$ significant difference compared to placebo (p < 0.05). p value between placebo and test product on $S$. epidermidis is 0.052 .

of beneficial bacteria ( $S$. epidermidis) to undesirable bacteria $(C$. striatum) $(\mathrm{p}=$ 0.03). Similarly, the Test Body Lotion with the triple biotic technology demonstrated a prebiotic effect in comparison to the Placebo Body Lotion by maintaining the growth of $S$. epidermidis, while inhibiting the growth of $S$. aureus (Figure 4(a) and Figure 4(b)). These results, expressed as $\log 10 \mathrm{CFU} / \mathrm{ml}$, show a statistically significant difference between the two lotions on $S$. aureus with the Test Body Lotion reducing the bacterial counts in comparison to the Placebo Body Lotion $(\mathrm{p}=0.048)$. There was no statistically significant difference between the two body lotions on the counts of $S$. epidermidis. As was seen with the Body Wash, the Test Body Lotion statistically significantly increased the ratio of beneficial bacteria (S. epidermidis) to deleterious bacteria $(S$. aureus $)(\mathrm{p}=0.029)$. These results are shown in Figure 4(c).

The effect of triple biotic technology on skin barrier property was evaluated in an ex vivo skin tissue model using dye penetration assay and skin barrier biomarkers measurement. Both the Test Body Wash and the Test Body Lotion, each with the Triple Biotic Technology, demonstrated an enhanced skin barrier (Figure 5) in the skin penetration assay. The amount of Rhodamine B fluorescence (red color) penetration into the skin is directly correlated with the barrier 
function. The better the barrier, the less dye that penetrates. In comparison to their respective placebos, there is little to no fluorescence dye that has penetrated into the skin for samples treated with either the Test Body Wash with Triple Biotic Technology or the Test Body Lotion with Triple Biotic Technology. The amount of fluorescence was quantified by Image Analysis, and the results are presented in Table 1. The Test Body Wash with the Triple Biotic Technology has produced statistically significantly less fluorescence than the Placebo Body Wash indicating much enhanced skin barrier $(\mathrm{p}<0.05)$. However, there was not a statistically significant difference in the amount of fluorescence produced by the Placebo Body Lotion and the Test Body Lotion with the Triple Biotic Technology.

Table 1. Intensity of Rhodamine B Fluorescence in skin tissues treated with body wash or body lotion with or without triple biotics technology as Measured by Image Analysis.

\begin{tabular}{cccc}
\hline & Mean $(\mathrm{n}=12)$ & Std Dev & p-value \\
\hline Placebo Body Wash & 0.17 & 0.27 & $<0.05$ \\
Body Wash with triple biotic technology & 0.0008 & 0.0029 & \\
Placebo Body Lotion & 0.34 & 0.45 & N.S. \\
Body Lotion with triple biotic technology & 0.12 & 0.14 & \\
\hline
\end{tabular}

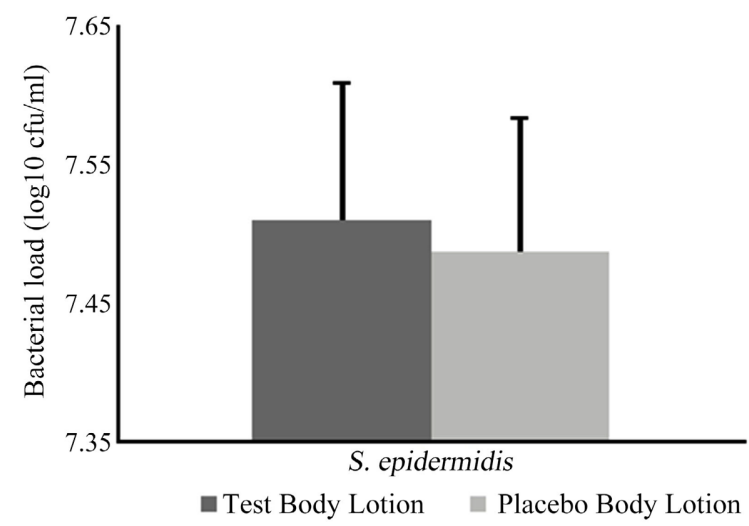

(a)

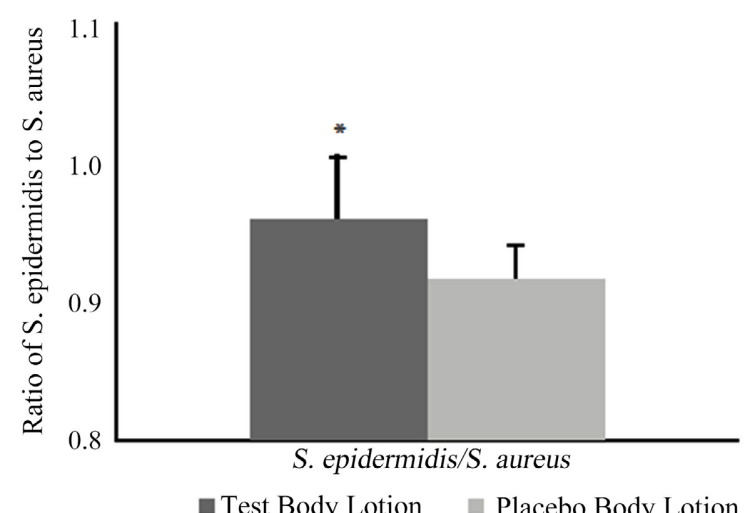

(c)

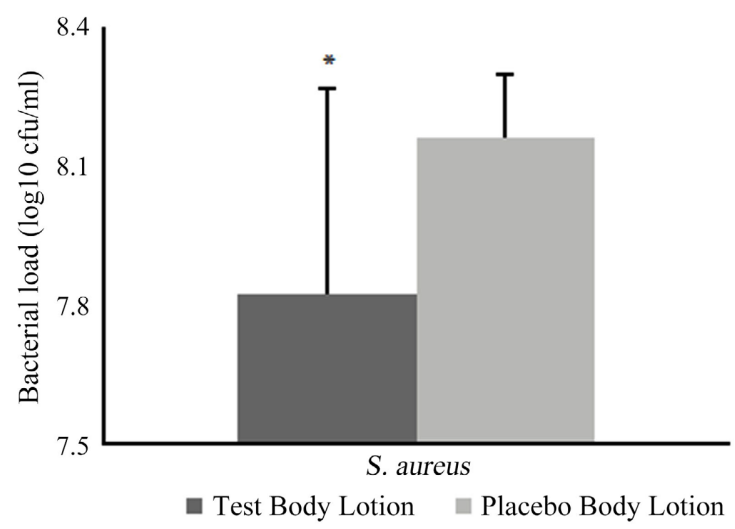

(b)

Figure 4. Comparison of Body Lotion with and without Triple Biotics Technology on the growth of $S$. epidermidis (4a), $S$. aureus. (4b), and the ratio of $S$. epidermidis/S. Aureus $(4 \mathrm{c}) .{ }^{*}$ significant difference compared to control $(\mathrm{p}<0.05)$. 


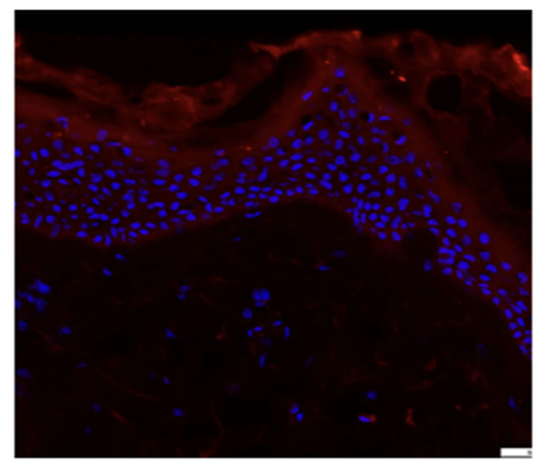

Placebo Body Wash

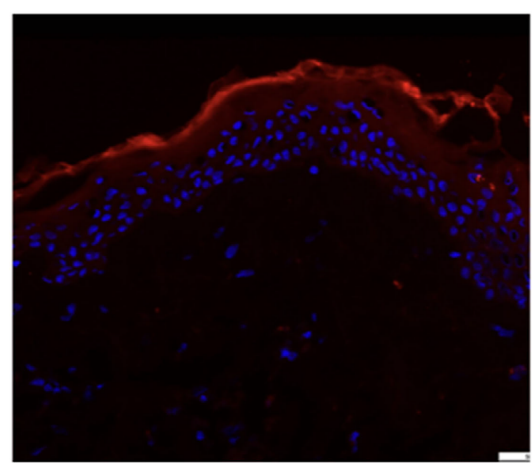

Placebo Body Lotion

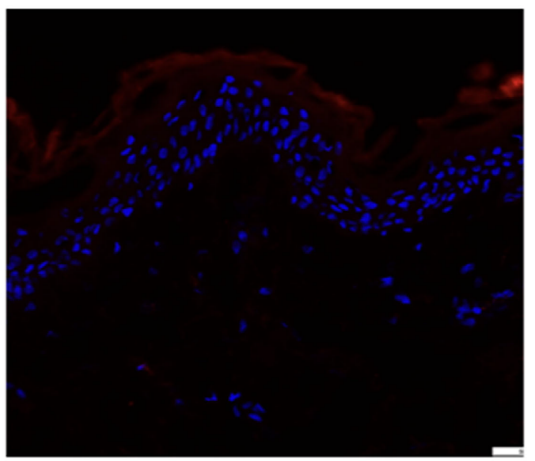

Test Body Wash

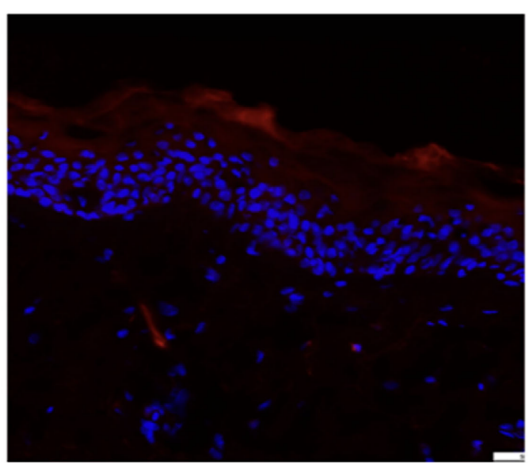

Test Body Lotion

Figure 5. Rhodamine B Fluorescence images of Skin tissues cross sections topically treated with body wash or body lotion with and without triple biotics technology. Red fluorescence is Rhodamine B, blue fluorescence is 4',6-diamidino-2-phenylindole (DAPI) fluorescence.

As shown in Figures 6(a)-(d), application of the Test Body Lotion with the Triple Body Technology has statistically significantly increased specific skin barrier biomarkers $(\mathrm{p}<0.05)$, namely, Filaggrin, Involucrin, Desmocollin 1 , and Claudin 1, in comparison to the Placebo Body Lotion. Quantitative comparisons were made between products by image analysis of the varying fluorescence levels, as described above for Rhodamine $\mathrm{B}$.

\section{Discussion}

Prebiotics, probiotics, and postbiotics are emerging technologies that are used in the personal care industry and have a beneficial effect on the skin microbiome [3] [4] [5] [6] [7] [16]. A new proprietary triple-biotic technology consisting of inulin, 2-butyloctanol, and a novel biomimic postbiotic blend has been developed and evaluated as individual components as well as when formulated into a body wash or body lotion on skin microbiome. We examine the effects on the promotion or inhibition of growth of four common skin bacteria. S. aureus, $C$. striatum, $E$. coli, and $S$. epidermidis were chosen as representatives for very specific reasons. $S$. epidermidis was chosen to represent the beneficial bacteria on the skin. Healthy skin is typically populated with coagulase negative staphylococci (CoNS) bacteria along with other bacteria [20] and S. epidermidis is the 


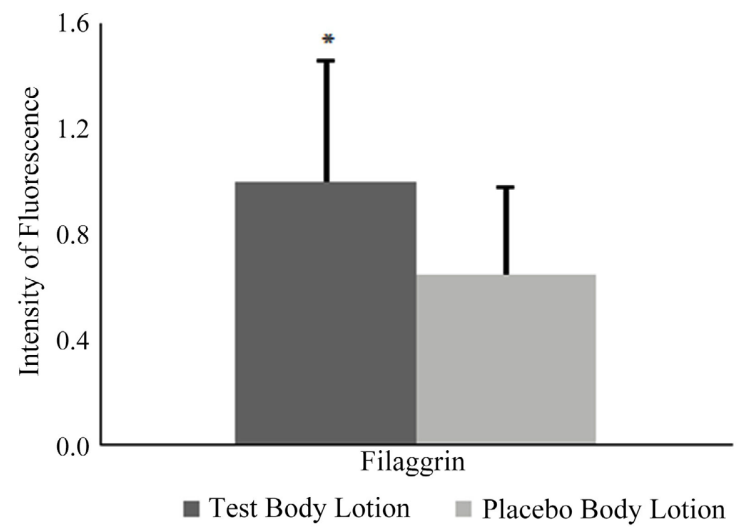

c

(a)

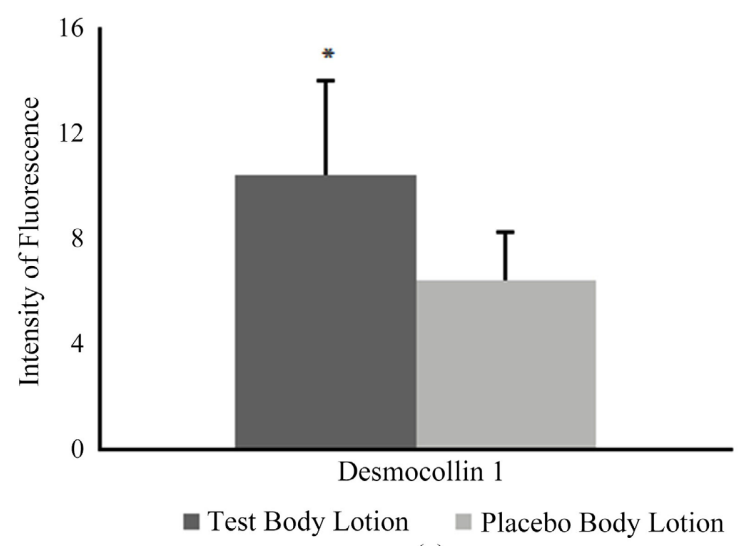

(c)

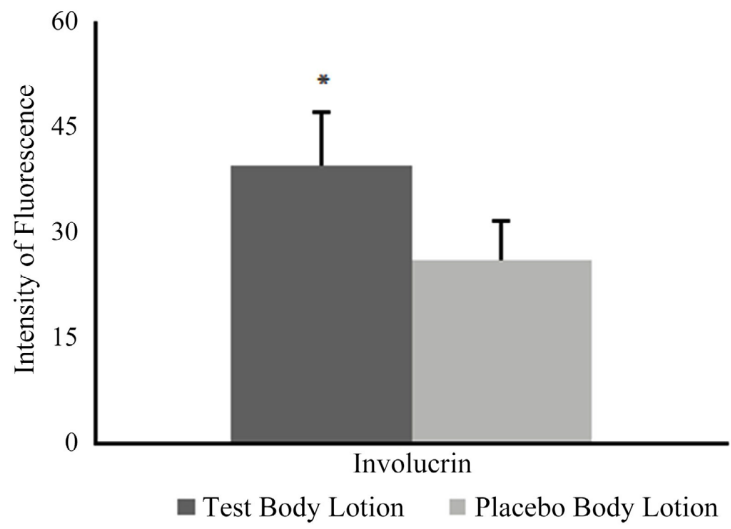

(b)

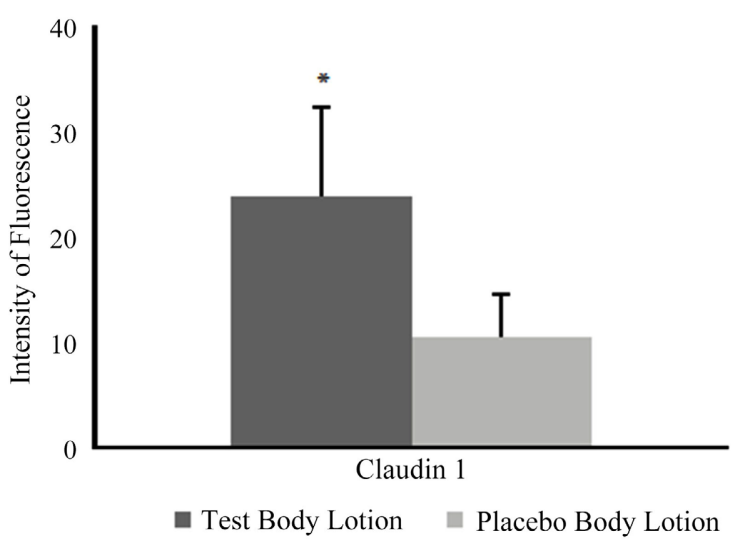

(d)

Figure 6. Effect of Body Lotions with and without Triple Biotics Technology on Skin Barrier Biomarkers: Filaggrin (a), Involucrin (b), Desmocollin 1 (c), and Claudin 1 (d). ${ }^{*}$ significant difference compared to control $(\mathrm{p}<0.05)$.

beneficial and frequently isolated species [21] [22]. It also can influence the colonization of $S$. aureus, which is one of the deleterious bacteria on the skin [20]. $S$. aureus is also a leading cause of skin infections and is well known for its involvement in atopic dermatitis [23] [24]. C. striatum is known to be an odor causing bacterium [25]. E. coli has been implicated in skin infections [26].

The first component of this technology, inulin, is a well-known prebiotics that has been widely used in intestinal health to modulate gut microbiome, but little is known with regards to its effect on the skin microbiome [9] [10] [11]. Here we demonstrated in Figure 1 that inulin has the ability to inhibit the growth of the undesirable bacteria ( $S$. aureus, C. striatum, E. coli) and maintain the growth of desirable bacteria ( $S$. epidermidis).

The second component, 2-butyloctanol, commonly used as an emollient in many skin care products, has been shown to have a prebiotic effect on human axillary microbiome [12]. Using both in vitro and in vivo studies, the authors determined that 2-butyloctanol inhibited odor causing corynebacteria while maintaining/promoting skin friendly $S$. epidermidis. The authors postulated that 2-butyloctanol could be incorporated into cosmetics and personal care products as a complex with other prebiotics, probiotics, and postbiotics. 
The third component of our technology, the biomimic blend has been designed to mimic the function of the lactobacillus ferment. According to a recent article, there are at least 50 cosmetic products that claim to contain probiotics. Of these, approximately $50 \%$ list lactobacillus ferment as one of the ingredients. The most common claims for these products are improving the skin barrier or "balancing" the skin microbiome [27]. Earlier research has demonstrated that an extract of lactobacillus has been shown to help repair the skin barrier when applied as an oil-in-water formulation [28]. It was also shown to reduce the skin microflora, but no individual bacterial species were identified. In our current study, two short chain acids, lactic acid and pyruvic acid were identified as main components in lactobacillus ferment and prepared in a 4:1 ratio. It is known that the salt of lactic acid, lactate, is a component of the skin's natural moisturizing factor (NMF) and can comprise up to $12 \%$ of NMF [29]. A study by Nakagawa et al. suggested that lactate may play a role in maintaining the physical properties of the stratum corneum leading to increased hydration [30]. More recently, the application of colloidal oat in a moisturizer was shown to increase lactic acid production and may help contribute to an improved skin condition in patients with compromised skin conditions [31]. Pyruvic acid is known for its exfoliation benefits [32] [33], but otherwise, there is not any information regarding its impact on the skin barrier. It is well known that lactic acid and pyruvic acid have antibacterial properties [34] [35]. To our knowledge, no data reported on the prebiotic effect of these two acids. In this study, we explored the prebiotic effect of the combination of lactic acid and pyruvic acid in a specific ratio by inhibiting the growth of the undesirable bacteria ( $C$. striatum and $E$. coli), while promoting the growth of desirable bacteria ( $S$. epidermidis).

In addition to the effect that the individual components have on the skin microbiome, the triple-biotic technology also has a beneficial effect on the skin microbiome when formulated into topical formulations. As noted by Puebla-Barragan and Reid, the use of probiotics, prebiotics, and microbiome by the cosmetic and personal care industry would be encouraging if there was strong scientific claims support and if mechanisms of action were elucidated [27]. We have done just this by identifying the impact of our technology on certain strains of skin bacteria. The recent publication by Liu-Walsh et al. evaluated a skin moisturizer containing $1 \%$ colloidal oat on skin microbes and demonstrated that it selectively increased the growth rate of $\mathcal{S}$. epidermidis, which may also be a major source of lactic acid in the skin [31]. Thus, it is believed that topical application of our triple biotic technology would not have a negative influence on $S$. epidermidis production based on our research and the publication by Liu-Walsh et al.

Topical applications of probiotic bacteria have the potential to enhance the natural barrier of the skin [3]. We evaluated the triple-biotic technology formulated into cosmetic formulations using an ex vivo skin penetration assay to determine the effect on skin barrier function. The observed decrease in skin fluorescence for the body wash formulation with the triple-biotic technology means 
a healthier, more intact skin with a better barrier. On the other hand the body lotion formulation produced a non-statistical decrease in fluorescence. Lack of statistical significance may be due to large variability in the data. Other researchers have also observed that topically applied probiotics can help repair skin barrier function [28] [36] [37] [38].

To further investigate the impact of the triple-biotic technology on skin barrier function, we followed the skin biomarkers, Filaggrin, Involucrin, Desmocollin 1, and Claudin 1, before and after topical application of the body lotion with the triple-biotic technology. Filaggrin is a protein that is known to be fundamental for the function and maintenance of the skin barrier [39] [40]. Involucrin is a protein that is involved with the formation of the cornified envelope of the skin and is crosslinked with keratin filaments along with other proteins [41]. Desmocollin 1 is a calcium dependent adhesion molecule (part of the cadherin family) [42] and is part of the main adhesive structure of the skin. Claudin 1 is another of the skin barrier proteins and has been shown to be less present in the skin of atopic dermatitis patients, who are known to have a poor barrier function, as compared to healthy control patients [43]. We observed statistically significant increases in all four skin biomarkers indicating an improved barrier function by applying a body lotion with triple biotic technology.

There are many products in the marketplace that may incorporate a single biotic or two into their cosmetic formulation to claim either skin or microbiome benefit, however, most of them didn't provide strong scientific evidence publicly. Together with our previous work of Li et al. [12], we have shown the combination of three biotic components delivered both microbiome and skin benefits in individual components as well as when introduced into cosmetic formulations.

\section{Conclusion}

A novel triple biotic technology has been developed and shown to have beneficial effects on the skin microbiome and skin barrier function through the use of in vitro and ex vivo studies. The benefits have been shown for the components both individually and when formulated into cosmetic formulations. Overall, this novel triple biotic technology was able to deliver a strong prebiotic effect and improve skin health in body wash and body lotion products and can be used as a desirable prebiotic ingredient in personal care products to provide skin health benefits.

\section{Acknowledgements}

The research was funded by the Colgate-Palmolive Company. Dr. Mack Morrison, President of Starfish Scientific Solutions was funded by Colgate-Palmolive Company to provide technical writing support.

\section{Conflicts of Interest}

The authors declare no conflicts of interest regarding the publication of this paper. 


\section{References}

[1] Gibson, G.R., Hutkins, R., Sanders, M.E., Prescott, S.L., Reimer, R.A., Salminen, S.J., Scott, K., Stanton, C., Swanson, K.S., Cani, P.D., Verbeke, K. and Reid, G. (2017) Expert Consensus Document: The International Scientific Association for Probiotics and Prebiotics (ISAPP) Consensus Statement on the Definition and Scope of Prebiotics. Nature Reviews Gastroenterology \& Hepatology, 14, 491-502. https://doi.org/10.1038/nrgastro.2017.75

[2] Sousa, V., Santos, E. and Sgarbieri, V. (2011) The Importance of Prebiotics in Functional Foods and Clinical Practice. Food and Nutrition Sciences, 2, 133-144. https://doi.org/10.4236/fns.2011.22019

[3] Al-Ghazzewi, F.H. and Tester, R.F. (2014) Impact of Prebiotics and Probiotics on Skin Health. Beneficial Microbes, 5, 99-107. https://doi.org/10.3920/BM2013.0040

[4] Bustamante, M., Oomah, B.D., Oliveira, W.P., Burgos-Díaz, C., Rubilar, M. and Shene, C. (2020) Probiotics Ad Prebiotics Potential for the Care of Skin, Female Urogenital Tract, and Respiratory Tract. Folia Microbiologica (Praha), 65, 245-264. https://doi.org/10.1007/s12223-019-00759-3

[5] Lolou, V. and Panayiotidis, M.I. (2019) Functional Role of Probiotics and Prebiotics on Skin Health and Disease. Fermentation, 5, Article No. 41. https://doi.org/10.3390/fermentation5020041

[6] Maguire, M. and Maguire, G. (2017) The Role of Microbiota, and Probiotics and Prebiotics in Skin Health. Archives of Dermatological Research, 309, 411-421. https://doi.org/10.1007/s00403-017-1750-3

[7] Ouwehand, A., Lahtinen, S. and Tiihonen, K. (2017) The Potential of Probiotics and Prebiotics for Skin Health. In: Farage, M.A., Miller, K.W. and Maibach, H.I., Eds., Textbook of Aging Skin, Springer, Berlin, 1299-1313. https://doi.org/10.1007/978-3-662-47398-6_77

[8] Nizioł-Łukaszewska, Z., Bujak, T., Wasilewski, T. and Szmuc, E. (2019) Inulin as an Effectiveness and Safe Ingredient in Cosmetics. Polish Journal of Chemical Technology, 21, 44-49. https://doi.org/10.2478/pjct-2019-0008

[9] Wilson, B. and Whelan, K. (2017) Prebiotic Inulin-Type Fructans and Galacto-Oligosaccharides: Definition, Specificity, Function, and Application in Gastrointestinal Disorders. Journal of Gastroenterology and Hepatology, 32, 64-68. https://doi.org/10.1111/jgh.13700

[10] Di Lodovico, S., Gasparri, F., Di Campli, E., Di Fermo, P., D’Ercole, S., Cellini, L. and Di Giulio, M. (2021) Prebiotic Combinations Effects on the Colonization of Staphylococcal Skin Strains. Microorganisms, 9, Article No. 37. https://doi.org/10.3390/microorganisms9010037

[11] Collins, S. and Reid, G. (2016) Distant Site Effects of Ingested Prebiotics. Nutrients, 8, Article No. 523. https://doi.org/10.3390/nu8090523

[12] Li, M., Truong, K., Pillai, S., Boyd, T. and Fan, A. (2021) The Potential Prebiotic Effect of 2-Butyloctanol on Human Axillary Microbiome. International Journal of Cosmetic Science. https://doi.org/10.1111/ics.12738

[13] Hill, C., Guarner, F., Reid, G., Gibson, G.R., Merenstein, D.J., Pot, B., Morelli, L., Canani, R.B., Flint, H.J., Salminen, S., Calder, P.C. and Sanders, M.E. (2014) Expert Consensus Document. The International Scientific Association for Probiotics and Prebiotics Consensus Statement on the Scope and Appropriate Use of the Term Probiotic. Nature Reviews Gastroenterology \& Hepatology, 11, 506-514. https://doi.org/10.1038/nrgastro.2014.66 
[14] Callewaert, C., Knödlseder, N., Karoglan, A., Güell, M. and Paetzold, B. (2021) Skin Microbiome Transplantation and Manipulation: Current State of the Art. Computational and Structural Biotechnology Journal, 19, 624-631. https://doi.org/10.1016/j.csbj.2021.01.001

[15] Wegh, C.A.M., Geerlings, S.Y., Knol, J., Roeselers, G. and Belzer, C. (2019) Postbiotics and Their Potential Applications in Early Life Nutrition and Beyond. International Journal of Molecular Sciences, 20, Article No. 4673. https://doi.org/10.3390/ijms20194673

[16] Guéniche, A., Bastien, P., Ovigne, J.M., Kermici, M., Courchay, G., Chevalier, V., Breton, L. and Castiel-Higounenc, I. (2010) Bifidobacterium longum Lysate, a New Ingredient for Reactive Skin. Experimental Dermatology, 19, e1-e8. https://doi.org/10.1111/j.1600-0625.2009.00932.x

[17] Peral, M.C., Martinez, M.A. and Valdez, J.C. (2009) Bacteriotherapy with Lactobacillus plantarum in Burns. International Wound Journal, 6, 73-81.

https://doi.org/10.1111/j.1742-481X.2008.00577.x

[18] Jeong, K., Kim, M., Jeon, S.A., Kim, Y.H. and Lee, S. (2020) A Randomized Trial of Lactobacillus rhamnosus IDCC 3201 Tyndallizate (RHT3201) for Treating Atopic Dermatitis. Pediatric Allergy and Immunology, 31, 783-792. https://doi.org/10.1111/pai.13269

[19] Gao, J., Li, Y., Wan, Y., Hu, T., Liu, L., Yang, S., Gong, Z., Zeng, Q., Wei, Y., Yang, W., Zeng, Z., He, X., Huang, S.H. and Cao, H. (2019) A Novel Postbiotic from Lactobacillus rhamnosus GG with a Beneficial Effect on Intestinal Barrier Function. Frontiers in Microbiology, 10, Article No. 477. https://doi.org/10.3389/fmicb.2019.00477

[20] Parlet, C.P., Brown, M.M. and Horswill, A.R. (2019) Commensal Staphylococci Influence Staphylococcus aureus Skin Colonization and Disease. Trends in Microbiology, 27, 497-507. https://doi.org/10.1016/j.tim.2019.01.008

[21] Christensen, G.J.M. and Brüggemann, H. (2014) Bacterial Skin Commensals and Their Role as Host Guardians. Beneficial Microbes, 5, 201-215.

https://doi.org/10.3920/BM2012.0062

[22] Brown, M.M. and Horswill, A.R. (2020) Staphylococcus epidermidis-Skin Friend or Foe? PLOS Pathogens, 16, e1009026. https://doi.org/10.1371/journal.ppat.1009026

[23] Becker, R.E. and Bubeck Wardenburg, J. (2015) Staphylococcus aureus and the Skin: A Longstanding and Complex Interaction. Skinmed, 13, 111-119.

[24] Geoghegan, J.A., Irvine, A.D. and Foster, T.J. (2018) Staphylococcus aureus and Atopic Dermatitis: A Complex and Evolving Relationship. Trends in Microbiology, 26, 484-497. https://doi.org/10.1016/j.tim.2017.11.008

[25] Fujii, T., Inoue, S., Kawai, Y., Tochio, T. and Takahashi, K. (2021) Suppression of Axillary Odor and Control of Axillary Bacterial Flora by Erythritol. Journal of Cosmetic Dermatology. https://doi.org/10.1111/jocd.14201

[26] Ranjan, A., Shaik, S., Nandanwar, N., Hussain, A., Tiwari, S.K., Semmler, T., Jadhav, S., Wieler, L.H., Alam, M., Colwell, R.R. and Ahmed, N. (2017) Comparative Genomics of Escherichia coli Isolated from Skin and Soft Tissue and Other Extraintestinal Infections. mBio, 8, e01070-17. https://doi.org/10.1128/mBio.01070-17

[27] Puebla-Barragan, S. and Reid, G. (2021) Probiotics in Cosmetic and Personal Care Products: Trends and Challenges. Molecules, 26, Article No. 1249.

https://doi.org/10.3390/molecules26051249

[28] Muizzuddin, N., Maher, W., Sullivan, M., Schnittger, S. and Mammone, T. (2012) Physiological Effect of a Probiotic on Skin. Journal of Cosmetic Science, 63, 385-395. 
[29] Hardings, C.R. and Rawlings, A.V. (2006) Effects of Natural Moisturizing Factor and Lactic Acid Isomers on Skin Function. In: Loden, M. and Maibach, H.I., Eds., Dry Skin and Moisturizers: Chemistry and Function, 2nd Edition, CRC Press, Boca Raton, 187-209.

[30] Nakagawa, N., Sakai, S., Matsumoto, M., Yamada, K., Nagano, M., Yuki, T., Sumida, Y. and Uchiwa, H. (2004). (2004) Relationship between NMF (Lactate and Potassium) Content and the Physical Properties of the Stratum Corneum in Healthy Subjects. Journal of Investigative Dermatology, 122, 755-763.

https://doi.org/10.1111/j.0022-202X.2004.22317.x

[31] Liu-Walsh, F., Tierney, N.K., Hauschild, J., Rush, A.K., Masucci, J., Leo, G.C. and Capone, K.A. (2021) Prebiotic Colloidal Oat Supports the Growth of Cutaneous Commensal Bacteria Including $S$. epidermidis and Enhances the Production of Lactic Acid. Clinical, Cosmetic and Investigational Dermatology, 14, 73-82. https://doi.org/10.2147/CCID.S253386

[32] Kontochristopoulos, G. and Platsidaki, E. (2017) Chemical Peels in Active Acne and Acne Scars. Clinics in Dermatology, 35, 179-182.

https://doi.org/10.1016/j.clindermatol.2016.10.011

[33] Berardesca, E., Cameli, N., Primavera, G. and Carrera, M. (2006) Clinical and Instrumental Evaluation of Skin Improvement after Treatment with a New 50\% Pyruvic Acid Peel. Dermatologic Surgery, 32, 526-531. https://doi.org/10.1111/j.1524-4725.2006.32106.x

[34] Wong, C., Chang, T., Yang, H. and Cui, M. (2015) Antibacterial Mechanism of Lactic Acid on Physiological and Morphological Properties of Salmonella Enteritidis, Escherichia coli and Listeria monocytogene. Food Control, 47, 231-236. https://doi.org/10.1016/j.foodcont.2014.06.034

[35] Chilicka, K., Rogowska, A.M., Szyguła, R., Dzieńdziora-Urbińska, I. and Taradaj, J. (2020) A Comparison of the Effectiveness of Azelaic and Pyruvic Acid Peels in the Treatment of Female Adult Acne: A Randomized Controlled Trial. Scientific Reports, 10, Article No. 12612. https://doi.org/10.1038/s41598-020-69530-w

[36] Capone, K., Kirchner, F., Klein, S.L. and Tierney, N.K. (2020) Effects of Colloidal Oatmeal Topical Atopic Dermatitis Cream on Skin Microbiome and Skin Barrier Properties. Journal of Drugs in Dermatology, 19, 524-531. https://doi.org/10.36849/JDD.2020.4924

[37] Butler, É., Lundqvist, C. and Axelsson, J. (2020) Lactobacillus reuteri DSM 17938 as a Novel Topical Cosmetic Ingredient: A Proof of Concept Clinical Study in Adults with Atopic Dermatitis. Microorganisms, 8, Article No. 1026. https://doi.org/10.3390/microorganisms8071026

[38] Jung, Y.-O., Jeong, H., Cho, Y., Lee, E.-O., Jang, H.-W., Kim, J., Nam, K.T. and Lim, K.-M. (2019) Lysates of a Probiotic, Lactobacillus rhamnosus, Can Improve Skin Barrier Function in a Reconstructed Human Epidermis Model. International Journal of Molecular Sciences, 20, Article No. 4289. https://doi.org/10.3390/ijms20174289

[39] Armengto-Carbo, M., Hernandez-Martin, A. and Torrelo, A. (2015) The Role of Filaggrin in the Skin Barrier and Disease Development. Actas Dermo-Sifiliográficas, 106, 86-95. https://doi.org/10.1016/j.adengl.2014.12.007

[40] Kezic, S. and Jakasa, I. (2016) Filaggrin and Skin Barrier Function. In: Agner, T., Ed., Skin Barrier Function: Current Problems in Dermatology, Vol. 49, Karger, Basel, 1-7. https://doi.org/10.1159/000441539

[41] Future, M. (2020) Regulation of Filaggrin, Loricrin, and Involucrin by IL-4, IL-13, IL-17A, IL-22, AHR, and NRF2: Pathogenic Implications in Atopic Dermatitis. 
International Journal of Molecular Sciences, 21, Article No. 5382. https://doi.org/10.3390/ijms21155382

[42] Garrod, D. and Chidgey, M. (2008) Desmosome Structure, Composition, and Function. Biochimica et Biophysica Acta-Biomembranes, 1778, 572-587.

https://doi.org/10.1016/j.bbamem.2007.07.014

[43] Batista, D.I., Perez, L., Orfali, R.L., Zaniboni, M.C., Samorano, L.P., Pereira, N.V., Sotto, M.N., Ishizaki, A.S., Oliveira, L.M., Sato, M.N. and Aoki, V. (2015) Profile of Skin Barrier Proteins (Filaggrin, Claudins 1 and 4) and Th1/Th2/Th17 Cytokines in Adults with Atopic Dermatitis. The Journal of the European Academy of Dermatology and Venereology, 29, 1091-1095. https://doi.org/10.1111/jdv.12753 